\section{Simple analysis of covariance: A BASIC program for microcomputers}

\author{
JOHN P. GALLA \\ Widener University, Chester, Pennsylvania
}

Simple analysis of variance is a useful and powerful statistical technique for testing the hypothesis of equal means. It allows for the comparison of two or more treatment samples, does not require equal ns for each treatment, and may be used with random or within-subject group assignment. Flexibility in subject group assignment is among its most useful features, since matched and repeated measures designs are often used to control for specific extraneous variables. Such direct experimental control often facilitates determination of significant differences in the criterion measure attributed to the treatment by providing a smaller estimate of experimental error.

For a variety of reasons, however, researchers are not always able to employ a within-group design to control for specific variables. If practical limitations prohibit such a design, but a particular variable should nevertheless be controlled, the researcher may employ the analysis of covariance. This procedure permits statistical, rather than experimental, control of variables in a manner similar to matching groups with respect to an extraneous variable.

Procedure. The analysis of covariance requires two observations for each subject. One is designated $X$ and is variously termed the concomitant, controlled, covariate, extraneous, or supplementary measure. This $\mathrm{X}$ variable is not of experimental interest to the researcher. The other variable is designated $\mathrm{Y}$ and represents the dependent or criterion variable.

The analysis of covariance takes into account the regression of the $\mathrm{Y}$ measures on the $\mathrm{X}$ measures. To the extent that an extraneous variable is correlated with the criterion variable, the covariance technique removes the influence of the extraneous variable through simple linear regression and thereby provides a smaller error variance than would be possible using simple analysis of variance. Moreover, like simple analysis of variance, simple covariance may be used with any number of samples and does not require equal observations for each treatment. This article describes a comprehensive yet easy to use BASIC program for simple analysis of covariance.

Program. The program automatically computes both a simple analysis of covariance and variance, a test for homogeneity of regression, means for the $\mathrm{X}$ and $\mathrm{Y}$ variables, and adjusted $Y$ means. It also provides a summary analysis of covariance table and presents elapsed processing time. After the computations are made, the user is given the option of computing any user-specified pairwise

The author's mailing address is: Social Science Division, Widener University, Chester, PA 19013.
Table 1

Sample Data for Simple Analysis of Covariance Treatment Groups

\begin{tabular}{|c|c|c|c|c|c|c|}
\hline \multicolumn{3}{|c|}{1} & \multicolumn{2}{|c|}{2} & \multicolumn{2}{|c|}{3} \\
\hline & $\underline{x}$ & $Y$ & & $Y$ & $x$ & $Y$ \\
\hline & 1 & 5 & 2 & 1 & 1 & 10 \\
\hline & 61 & 12 & 3 & 2 & 4 & 13 \\
\hline & 3 & 9 & 6 & 7 & 5 & 16 \\
\hline & 4 & 8 & 4 & 3 & 3 & 12 \\
\hline & $\underline{5}$ & $\underline{11}$ & $\underline{Z}$ & $\underline{\mathbf{B}}$ & $\underline{\underline{b}}$ & $\underline{17}$ \\
\hline Sum 1 & 19 & 45 & 22 & 21 & 19 & 68 \\
\hline
\end{tabular}

Note-Sample data are from Edwards (1972, p. 373).

comparisons of means. The program is based on a variety of published statistical procedures for analysis of covariance (Edwards, 1972; Ferguson, 1976; Roscoe, 1975; Winer, 1962) and has been tested for accuracy with sample data from all referenced sources.

All computations are made with single-precision (sixdigit) accuracy. However, answers are rounded back to three decimal places by formatting output with PRINT USING statements. This format allows for accuracy within the limits presented in the referenced sources while still providing manageable screen and hardcopy output.

Input. The program limits input to $\mathrm{N}=100$ treatment groups with 50 observations per $\mathrm{X}$ and $\mathrm{Y}$ variable. The user can alter this limitation by redimensioning the variables in line 370 .

Data are read from DATA statements beginning in line 1000 . The first DATA statement contains $N$, the number of treatments or levels of the independent variable. Subsequent DATA statements begin with $\mathrm{n}$, the number of pairs of observations for a particular treatment, followed by the score data in pairs for that treatment. Table 1 provides sample data for the analysis, and Table 2 provides an example of how the DATA statements are set up using the sample data.

The program asks the user if instructions are required. If the answer is "yes," instructions on how to place the data into DATA statements, and how to receive a listing of the data, are given. If the answer is "no," the program assumes data have been entered and computes the analysis. If no new data have been entered, analysis of the sample data is performed.

Table 2

Sample Data Statements for Simple Analysis of Covariance

1000 DATA 3

1010 DATA $5,1,5,6,12,3,9,4,8,5,11$

1020 DATA $5,2,1,3,2,6,7,4,3,7,8$

1030 DATA 5. 1.10.4.13.5.16.3.12.6.17 
Table 3

Sample Output

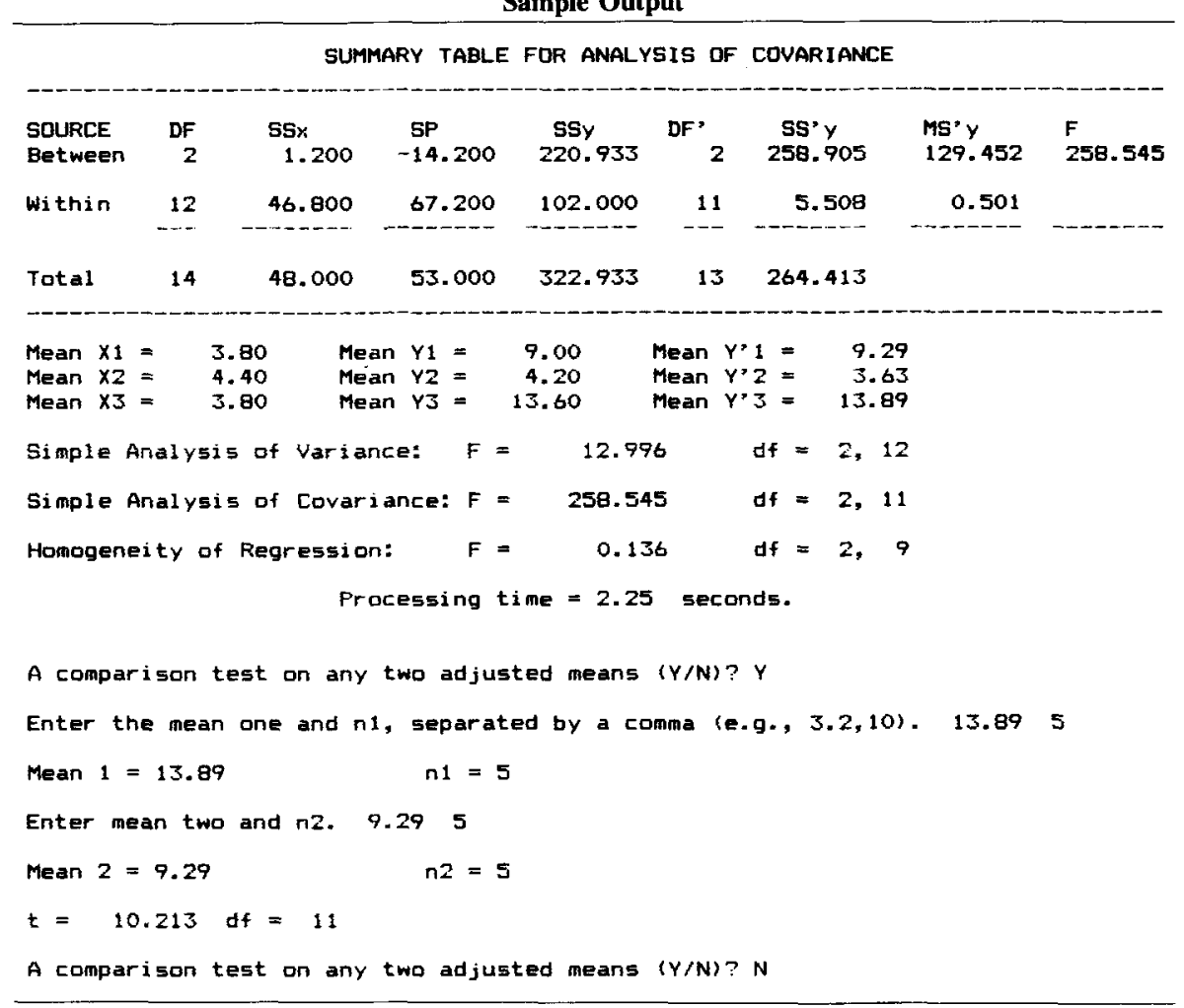

Output. Output is automatically directed to the display. However, the program asks the user whether or not output should also be sent to a printer. Sample output is presented in Table 3.

Program Language and Requirements. The program is written in IBM PC BASICA. It consists of 133 lines, including REMark and DATA statements, and uses approximately $6 \mathrm{~K}$ bytes of RAM. No essential IBM-specific functions are included, so that the program is easily ported to other microcomputer BASICs. The program may be easily modified to read from INPUT statements or data files.

Availability. A listing of the program may be obtained by writing to the author. A disk copy may be obtained by sending a double-sided/double-density diskette in a re- usable diskette mailer. Program users with suggestions or questions about the program may call the author at (215)499-7373.

\section{REFERENCES}

EDWARDS, A. L. (1972). Experimental design in psychological research (4th ed.). New York: Holt, Rinehart \& Winston.

FERGUSON, G. A. (1976). Statistical analysis in psychology and education (4th ed.). New York: McGraw-Hill.

RosCOE, J. T. (1975). Fundamental research statistics for the behavioral sciences (2nd ed.). New York: Holt, Rinehart \& Winston.

Winer, B. J. (1962). Statistical principles in experimental design. New York: McGraw-Hill.

(Revision accepted for publication December 11, 1984.) 